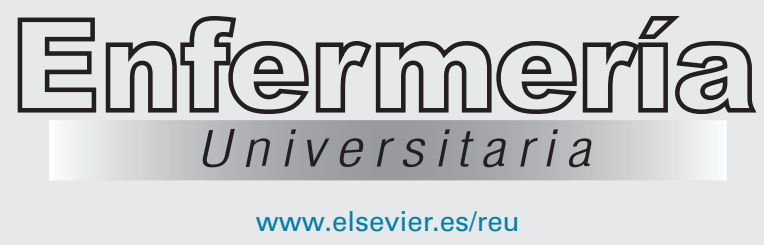

EDITORIAL

\title{
La Enfermería de Práctica Avanzada ¿qué es? y ¿qué podría ser en América Latina?
}

\section{Advanced Practice Nursing: What is it and what could it become in Latin America?}

\section{A enfermagem de prática avançada, o que é? e que poderia ser na América Latina?}

En los últimos 4 años se han desarrollado diversas acciones de la Organización Panamericana de la Salud (OPS) y de algunos de sus Centros Colaboradores, con apoyo de la Asociación Latinoamericana de Escuelas y Facultades de Enfermería (ALADEFE), con el propósito de hacer un análisis relativo al movimiento denominado Enfermería de Práctica Avanzada (EPA), que se originó en los estudios formales de la Enfermería a nivel de posgrado, al menos 60 años atrás en algunos países de nuestro continente, principalmente en Estados Unidos y Canadá, como una estrategia que ha incrementado el acceso a los servicios de salud y la cobertura en salud desde la perspectiva de la Atención Primaria a la Salud (APS), pero que también muestra evidencia de una fuerte tendencia de crecimiento en los sistemas de atención a la salud en todo el mundo ${ }^{1}$.

Los análisis de dicha temática se han profundizado en 2 reuniones que la OPS en conjunto con otras organizaciones realizaron en 2015 y 2016 en los Colegios de Enfermería de la Universidad de McMaster y la Universidad de Michigan, respectivamente, lo que culminó con la conformación de un grupo técnico que actualmente trabaja en la elaboración del reporte de un estudio de consenso que tuvo como propósito conocer la percepción de enfermeras de los sectores de Salud y Educación, relativo a las Competencias centrales que debe tener una enfermera de práctica avanzada contextualizadas a la perspectiva de América Latina.

Dichas acciones han tenido como propósito la construcción de una plataforma que permita que la EPA pueda ser impulsada de manera más sistemática en América Latina y

La revisión por pares es responsabilidad de la Universidad Nacional Autónoma de México. el Caribe, ya que a menudo se puede observar en los distintos países que el personal de enfermería en zonas rurales proveen servicios a poblaciones menos atendidas, en los que desempeñan un rol de práctica avanzada, aún falta el desarrollo formal de aptitudes, funciones claramente definidas y formación a nivel de posgrado que posibilite el nivel de autonomía requerido ${ }^{2}$.

Según Hutchinson et al., en los últimos años numerosos estudios han examinado a nivel mundial las características de la EPA, no obstante se han encontrado dificultades derivadas de los diferentes términos que utilizan para referirse a tipos similares de actividad, lo que también ha creado problemas en la investigación para medir su impacto y eficacia. Aunque de manera general la EPA puede ser caracterizada como el uso del conocimiento experto, habilidades complejas para tomar decisiones y desarrollo de competencias clínicas para la práctica de rol ampliado dirigido a poblaciones específicas, todavía se puede identificar la falta de acuerdo sobre qué dominios y actividades prácticas proporcionan una descripción exacta y significativa de lo que es la $\mathrm{EPA}^{3}$.

El metaanálisis de estos autores reveló 7 dominios distintos de práctica y uno relacionado con la práctica «estándar» de la Enfermera registrada (RN por sus siglas en inglés).

Los 7 dominios de la práctica avanzada que identificaron fueron: práctica clínica ampliada autónoma o liderada por la enfermera, desarrollo de la práctica de otros, mejora de los sistemas de cuidado, desarrollo y oferta de programas y actividades educativas, así como diseño y liderazgo de investigación y empleo de los resultados de investigación en la práctica. 
Por su parte, el Consejo Internacional de Enfermería ha definido a la enfermera de práctica avanzada como una enfermera que ha logrado una base de conocimiento experto, habilidades para la toma de decisiones clínicas complejas y competencias para ejercer un papel extendido; características que son delineadas de acuerdo al contexto del país en el que ha sido licenciada para ejercer profesionalmente, por lo cual se considera que su nivel de entrada debe ser la Maestría.

$Y$ ha señalado que la naturaleza de dicha práctica implica:

- Un alto grado de autonomía y ejercicio profesional independiente.

- El manejo de casos a través de poner en práctica competencias clínicas avanzadas reconocidas: para la valoración, el razonamiento diagnóstico y la toma de decisiones.

- Proveer servicios de consultoría a proveedores de salud.

- Desarrollar planes, implementación y evaluación de programas.

- Ser el primer punto de contacto reconocido para clientes ${ }^{4}$.

Como se puede observar son roles diferentes a los que de forma tradicional realiza una enfermera con formación general, e involucra necesariamente, además de la formación, una serie de factores a nivel macro que necesitan ser considerados si se quiere implantar en cada país como un modelo de atención.

Todo lo anterior sin duda apoyaría la calidad de la atención a la salud y de las prácticas del cuidado de Enfermería, así como lograr mayor acceso y cobertura universal en salud, optimizar la utilización de recursos de Enfermería (especialistas, maestros y doctores en Enfermería), con énfasis en la práctica avanzada en Enfermería, asimismo hacer visible la contribución de la Enfermería en la atención a la salud.

Dentro de los principales análisis que se han hecho en la literatura están, por un lado, los motivos que promovieron el origen de la EPA, que estuvo referida principalmente al déficit de médicos, áreas con falta de servicios de salud o con poco equipamiento y a las características geográficas de algunos países con la consecuente dispersión de las poblaciones humanas. Como ejemplo se puede citar el caso de Canadá, que desde finales del siglo XIX y primera mitad del siglo $x x$ comenzó a vivenciar, aunque en contextos no formales, la EPA. A partir de 1960 se comenzó con la educación formal de la Nurse practitioner ${ }^{1}$ y las Clinical Nurse Specialist (enfermeras clínicas especialistas). Todavía, en la actualidad casi el $20 \%$ de su población vive en comunidades remotas y aisladas, particularmente en el norte y noreste del país ${ }^{5}$.

\footnotetext{
${ }^{1}$ A diferencia de los países anglosajones, en la mayoría de los países de América Latina no se tiene una figura equivalente a la Nurse practitioner y aunque la traducción literal sería Enfermera practicante, se trata más bien de una enfermera cuya formación de posgrado le permite desempeñar la enfermería asumiendo un rol mucho más amplio que el tradicional, como ya ha sido señalado en este mismo documento.
}

Numerosas controversias existieron en su momento respecto a que las enfermeras se hicieran cargo de la atención de dichas poblaciones. Algunas estaban referidas a que de esta manera las enfermeras tendrían los conocimientos y habilidades para desarrollar un rol expandido de Enfermería, y otras a la posible «medicalización» de la Enfermería.

En ese sentido, en el caso de América Latina, ya existen grupos que en distintos países realizan foros de discusión respecto a las controversias previsibles de la aplicación de la EPA, de tal forma que desde el principio se identifiquen o generen los marcos conceptuales que den sustento a este movimiento. Las evidencias de experiencias, buenas prácticas y lecciones aprendidas de otras regiones, dimensionándolas en el contexto propio, pueden ser buenos referentes y apoyar un desarrollo más rápido.

Como se señaló con anterioridad, también en la literatura han sido analizados el o los roles que puede desempeñar una enfermera que realiza EPA, que en principio ha sido compromiso de las Nurse Practitioner y de las Enfermeras clínicas especialistas, enfermeras reconocidas en Estados Unidos y Canadá, como profesionales que han tenido impacto en el aumento del acceso a la APS y que han mejorado la calidad de la atención y los resultados de salud ${ }^{6}$.

La APS es una actividad clave de la EPA, ya que conjunta las actividades mencionadas previamente para encargarse del cuidado de la salud de las comunidades, incluso la enfermería ha sido reconocida como la profesión que cuenta con más integrantes dedicados al cuidado de la salud en los Estados Unidos, porque la educación que reciben les permite valorar el estado de salud de los pacientes, proveer tratamiento clínico y educar a los pacientes y familias sobre la prevención de enfermedades y el mantenimiento de su salud, a través de la valoración y reducción de riesgos en los individuos y comunidades; imparte educación para la salud, brinda asesoría a pacientes, familias y grupos, maneja enfermedades crónicas y asiste a pacientes y familias para interpretar y valorar información de salud aunado al uso de tecnologías ${ }^{7}$. Los roles profesionales de enfermería son un amplio campo de oportunidad para el ejercicio de la EPA y tienen un amplio reconocimiento social, por medio de su práctica pueden hacer grandes contribuciones cuando se conocen las necesidades primarias, secundarias y terciarias de cuidado de su país.

El modelo de roles a desempeñar en la EPA se centra principalmente en conocer las necesidades de salud del paciente o grupos, como vía para aplicar al máximo el conocimiento y habilidades de la enfermera, en pro de la mejora, tanto en los servicios de enfermería como en los de salud. También incluye la colaboración con los médicos y otros profesionales de la salud, determinar los mecanismos de interacción entre ellos, definir la visión de práctica, el establecimiento de normas y el desarrollo de protocolos, proveer programas educativos, evaluar el impacto y determinar las necesidades y momento más oportuno del involucramiento de los distintos proveedores de salud ${ }^{8}$.

Es decir, la enfermera de práctica avanzada promueve intervenciones directas de cuidado avanzado basadas en una valoración integral del paciente que le permiten realizar diagnósticos diferenciales, emprender la gestión de casos, proporcionar intervenciones terapéuticas complejas o avanzadas, ser guía clínica profesional que brinda apoyo, consultoría y tutoría a los estudiantes de enfermería y de 
otras disciplinas del área de salud y al mismo equipo profesional multidisciplinario, contribuir a la mejora de los sistemas de salud, a través del desarrollo de nuevos servicios o programas de salud, así como el desarrollo e impartición de programas educativos y también realizar y fomentar la investigación ${ }^{3}$.

Existen muchas evidencias que muestran también ejemplos de aplicación de ese rol extendido o ampliado de la Enfermería en otros niveles de atención, no solamente en la APS, que están reportadas en la literatura.

Por ejemplo, en la Facultad de Enfermería de la Universidad de los Andes en Chile, el énfasis que le han dado a la Maestría en EPA es en la atención del Paciente Crítico Adulto, que busca formar en la enfermera competencias avanzadas en el cuidado del paciente adulto con enfermedad aguda, que se encuentre en áreas críticas.

Además del contexto anglosajón, en los países escandinavos y los Países Bajos, y seguramente en países de otros continentes, así como en España, se pueden observar diversas experiencias, por ejemplo: la gestión de casos en Andalucía, las estrategias frente a la cronicidad compleja en el País Vasco, o la atención urgente en atención primaria de Cataluña.

Sastre-Fullana et al. en el 2015 en España realizaron un estudio para contrastar la validez de contenido de un conjunto de competencias desarrolladas en distintos contextos a nivel mundial, para el ejercicio de la práctica avanzada en enfermería. Lograron identificar competencias comunes en la EPA que se agruparon bajo distintos dominios conceptuales, asimilables a los roles de práctica avanzada en enfermería, dentro de su contexto inmediato de práctica profesional.

Identificaron los siguientes dominios: investigación y práctica basada en evidencia, liderazgo clínico, consultoría y tutoría, colaboración y relación interprofesional, juicio clínico experto, liderazgo educativo, gestión de calidad y seguridad clínica del cuidado, autonomía para la práctica profesional, promoción de la salud, competencia cultural y protección y defensa de los derechos del paciente.

De dichos dominios despuntaron 4: liderazgo clínico, investigación, práctica basada en evidencia y gestión del cuidado, que denotan de alguna manera mayor especificidad para enfermeras de práctica avanzada. Acorde a lo que Gardner en Australia encontró utilizando el Strong Model of Advanced Practice Role Delineation Tool, ya que había importantes diferencias en los distintos dominios de competencias entre las enfermeras que ejercían roles de EPA y las que no ${ }^{9}$.

Un elemento esencial para la preparación de las enfermeras de práctica avanzada son los procesos de formación, que como se ha señalado es deseable se inicie en el posgrado, sin embargo sería muy importante como estrategia iniciar y desarrollar progresivamente desde el pregrado conocimientos, habilidades, actitudes y valores que vayan conformando un perfil que apoye la oferta de cuidado de calidad, pero que a la vez apoye el desarrollo y profundización posterior en los estudios de posgrado de EPA.

Respecto a la educación en enfermería la National Organization of Nurse Practiotioner Faculties (NONPF) y la American Association of Colleges of Nursing (AACN) de los Estados Unidos sugirieron un modelo consensuado para formar a las Nurse Practitioners, en el que estipularon que los programas de educación deben incluir como mínimo 3 niveles de cursos comprensivos: fisiología/patofisiología avanzada (involucra principios generales aplicables a la esperanza de vida), valoración avanzada de la salud (valoración de los sistemas humanos, técnicas de valoración avanzada, conceptos y aproximaciones), y farmacología avanzada (farmacodinamia, farmacocinética y farmacoterapéutica en todas sus categorías de agentes) ${ }^{10}$.

El modelo pretende que las enfermeras desarrollen las competencias: Fundamentación científica, Liderazgo, Calidad, Consulta, Uso práctico de la Tecnología de Información y comunicación, Política, Asesoramiento de los Sistemas de Salud, Ética y Práctica Independiente.

Es significativo señalar que para el avance de la EPA no solamente se deben analizar los factores de origen en los países, el establecimiento de roles a desempeñar y de las capacidades a desarrollar a través de la educación formal y continua, sino que tendrían necesariamente que ser promovidos de manera simultánea cambios que permitan incidir en las políticas públicas de trabajo, economía, educación y salud; la regulación de la educación y de la práctica, la estructura y normatividad de los sistemas de atención a la salud y en consecuencia de sus modelos de atención, los sistemas de planificación de recursos humanos en salud, el empoderamiento de la sociedad civil y los sistemas de acreditación de calidad de establecimientos y la certificación del personal de salud.

Es decir, si bien la formación de las enfermeras de práctica avanzada es un elemento central, para lograr implementar de manera formal dicho movimiento en los países de América Latina, no se puede pensar exclusivamente en la formación de sus competencias. De forma simultánea se necesita trabajar en otros frentes y niveles, por ejemplo: incurrir en los órganos legislativos y normativos; con los tomadores de decisiones en materia de salud y educación, incluidas las enfermeras gubernamentales; con los planificadores de los sistemas y recursos humanos en salud; con grupos de la sociedad civil, incluidos en estos las organizaciones de pacientes; con los organismos nacionales e internacionales de acreditación y regulación de la calidad, entre otros.

América Latina es un gran mosaico heterogéneo en lo político, económico, educativo y cultural. El análisis que se ha iniciado al interior de los países de América Latina respecto a la EPA representa una buena estrategia, ya que posibilita, de manera contextualizada, realizar análisis con un enfoque más integral respecto a la realidad de los elementos ya referidos, que inciden directamente sobre el avance que esta pueda tener, así como de las fortalezas y las áreas de oportunidad que existen en los diversos países para su desarrollo y avance.

Quizá deberíamos preguntarnos en primer lugar, ¿cuáles son las circunstancias de nuestro país respecto al movimiento de EPA? Porque nunca se ha pensado que el desarrollo de la EPA en Australia, o en Estados Unidos, o en Canadá, o en España, o en Holanda, sea como una figura cuadrada perfecta y que lo que queremos es lograr construir o reconstruir lo que sería la EPA en los países de América Latina a total semejanza, como otra figura cuadrada que embonara perfecto. Eso sería irreal y ahistórico. Pero esto no significa que no debamos aprovechar las buenas prácticas y las lecciones aprendidas de experiencias de otros países, 
ya que eso nos permitiría dar pasos más sólidos y acortar caminos, si se toman en consideración evidencias de experiencias anteriores, siempre enmarcadas en los contextos particulares.

Lo que debemos hacer también es un diagnóstico de cuáles son las prácticas que ya se llevan a cabo en nuestro país, que son claros ejemplos de EPA en tanto que están dando respuesta a necesidades sociales en materia de salud, particularmente en APS, que son una clara expresión de cómo ha sido conceptualizada la EPA, que buscan lograr prácticas del cuidado de calidad y por lo tanto mejores resultados, que representan ejemplos de la autonomía y visibilidad de la práctica enfermera. Seguramente nos sorprenderíamos al identificar que existen experiencias en nuestros países, tal vez con diferentes estadios de desarrollo, pero ello nos permitiría identificar los caminos a seguir para consolidarlas, o para ofrecerles estrategias que apoyen su avance.

Es altamente probable que muchas de ellas, aun consolidadas, no hayan sido documentadas y publicadas, lo que finalmente hace que no se cuente con un inventario de experiencias exitosas y no exitosas, que no haya referentes para medición de su impacto y efectividad, pero sobre todo, que se pierda visibilidad ante la comunidad científica internacional.

Bryant-Lukosius et al. han hecho recientemente un análisis de algunas experiencias desarrolladas en América Latina y el Caribe, así como de la situación en que está el desarrollo de los estudios de posgrado, en países con ingresos medios y altos por lo que consideran estarían posicionados para introducir los roles de la EPA ${ }^{11}$.

Quizá un problema sustancial para el avance de la EPA es la regulación de la educación y la práctica de la Enfermería, en cuyo caso se requiere que cada vez más las enfermeras participen en posiciones que permitan la toma de decisiones, pues aunque tenemos en América Latina buenos ejemplos de enfermeras gubernamentales y del avance de sus proyectos a nivel nacional, se requiere que además de la facultad de emitir recomendaciones o normas puedan tener facultades decisorias.

En ese sentido, algunas preguntas por resolver podrían ser ¿De qué manera la estructura actual de los servicios de salud en los diversos países da cabida a un profesional de Enfermería para la EPA?, ¿inmersos en qué políticas?, ¿en qué escenarios sería más necesario y factible implementarlos?, ¿cuáles serían los recursos disponibles?, ¿para qué modelos de atención?, ¿con qué nivel de desarrollo del trabajo interprofesional?, ¿con qué condiciones laborales?

Seguramente, las respuestas se estarán generando en la medida que grupos formales de expertos de nuestros países asuman el compromiso de analizar la situación nacional actual y la perspectiva para lograr el avance de la EPA, así como el planteamiento de un plan estratégico que apoye su desarrollo, de tal forma que, como lo muestran las evidencias, se convierta en un movimiento nacional que mejore el acceso a la atención de la salud de calidad con mayor cobertura.

Como conclusión, podemos afirmar que como región y países tenemos grandes desafíos:

- Hacer un diagnóstico e inventario de experiencias exitosas y lecciones aprendidas por país.
- Definir los roles a desempeñar en la EPA, dominios, y capacidades integrales para desempeñarla.

- Incrementar el trabajo colaborativo real y efectivo entre educación y asistencia, a través de las Comisiones Nacionales de Enfermería.

- Promover cambio de paradigmas en los diseños curriculares que apoyen transformaciones curriculares en pro de la formación avanzada, tanto en el pregrado como en el posgrado.

- En tanto nos incorporamos a los órganos decisorios, necesitamos aprender a incidir políticamente con los tomadores de decisiones.

- Trabajo colaborativo sostenido entre organismos nacionales e internacionales.

- Transformación de los sistemas y modelos de atención a la salud, particularmente de la atención primaria, con participación de otros líderes intersectoriales.

- Involucramiento de las enfermeras gubernamentales para que aceleren los procesos legislativos y de regulación de la práctica avanzada en Enfermería.

Todas las acciones que emprendamos en ese sentido, sin importar la posición que ocupemos, deberán favorecer una alineación de los roles de EPA con la agenda global, para ampliar la cobertura universal de salud y el acceso a la misma, lo cual además será una clara expresión de la responsabilidad social que tenemos como gremio de Enfermería, para poner al servicio de individuos y colectivos prácticas avanzadas de Enfermería a través del cuidado seguro y de calidad.

\section{Referencias}

1. Sheer B, Wong FK. The development of Advance Practice Nursing Globally. J Nurs Scholars. 2010;40:204-11, http://dx.doi.org/ 10.1111/j.1547-5069.2008.00242.x

2. Cassiani S, Zug KE. Fomentando el papel de la Práctica Avanzada de Enfermería en América Latina. Rev Bras Enferm. 2014;67:677-8. http://dx.doi.org/10.1590/0034-7167.201467 0501

3. Hutchinson M, East L, Stasa $\mathrm{H}$, et al. Deriving consensus on the characteristics of Advanced Practice Nursing. Meta-summary of more than 2 decades of research. Nurs Res. 2014;62:116-28, http://dx.doi.org/10.1097/NNR. 0000000000000021

4. International Council of Nurses. Definition and characteristics for nurse practitioner/advanced practice nursing roles. Geneva, Switzerland: ICN, 2002 [citado 6 Sep 2017]. Disponible en: www.icn.ch/es.

5. Bryant-Lukosius D, Valaitis R, y Faculties from Canadian Centre for APN Research (CCAPNR). Canadian Report Global. Presentado en el Advance Practice in Nursing Summit, organizado por la OPS. 28-29 de julio de 2014.

6. Martin-Misener R, Harbman P, Donald F, et al. Cost-effectiviness of nurse practitioners in primary and specialized ambulatory care: Systematic review. BMJOpen. 2015:e007167. http:// dx.doi.org/10.1136/bmjopen-2014-007167

7. Zenzano T, Allan JD, Bigley MB, et al. The roles of healthcare professionals in implementing clinical prevention and Ppopulation health. Am J Prev Med. 2011;40:261-7, http://dx.doi.org/ 10.1016/j.amepre.2010.10.023

8. Bryant-Lukosius B, DiCenso A. A framework for the introduction and evaluation of advanced practice nursing roles. $J$ Adv Nurs. 2004;48:530-40, http://dx.doi.org/10.1111/j.13652648.2004.03235.x 
9. Sastre-Fullana P, de Pedro-Gómez JE, Bennasar-Veny M, et al. Consenso sobre competencias para la enfermería de práctica avanzada en España. Enferm Clin. 2015. http://dx.doi.org/ 10.1016/j.enfcli.2015.06.007

10. National Organization of Nurse Practitioner Faculties. Population-Focused Nurse Practitioner Competencies. National Organization of Nurse Practitioner Faculties. 2013. p. 9-20.

11. Bryant-Lukosius D, Valaitis R, Martin-Misener R, et al. Advanced Practice Nursing: A strategy for achieving universal health coverage and universal access to health. Rev. Latino-Am.
Enfermagem. 2017;25:e2826, http://dx.doi.org/10.1590/15188345.1677 .2826

L. Morán-Peña

Presidenta de la Asociación Latinoamericana de Escuelas y Facultades de Enfermería, Ciudad de México, México Escuela Nacional de Enfermería y Obstetricia, Ciudad de México, México Correo electrónico: eneolm@yahoo.com 